\title{
Accelerated Aqueous Solubility and Antibacterial Activity of Cefuroxime Axetil Using Microcrystalline Cellulose as Carrier
}

\author{
Moushumi Tabassoom Salam1, Ashim Kumar1, Akito Hata², Hiromu Kondo², Md. Abdus Salam³, \\ Mir Imam Ibne Wahed1, Md. Rafiqul Islam Khan', Ranjan Kumar Barman ${ }^{1 *}$ \\ ${ }^{1}$ Laboratory of Pharmaceutics, Department of Pharmacy, Faculty of Science, University of Rajshahi, Rajshahi, Bangladesh \\ ${ }^{2}$ Laboratory of Pharmaceutical Engineering \& Drug Delivery Science, School of Pharmaceutical Science, University of Shizuoka, \\ Shizuoka, Japan \\ ${ }^{3}$ Department of Basic Medical Sciences, Faculty of Medicine, International Islamic University Malaysia, Kuantan, Malaysia \\ Email: ${ }^{\star d r b a r m a n 76 @ g m a i l . c o m ~}$
}

How to cite this paper: Salam, M.T., Kumar, A., Hata, A., Kondo, H., Salam, Md.A., Wahed, M.I.I., Khan, Md.R.I. and Barman, R.K. (2020) Accelerated Aqueous Solubility and Antibacterial Activity of Cefuroxime Axetil Using Microcrystalline Cellulose as Carrier. Pharmacology \& Pharmacy, 11, 159-173.

https://doi.org/10.4236/pp.2020.118015

Received: July 2, 2020

Accepted: August 9, 2020

Published: August 12, 2020

Copyright $\odot 2020$ by author(s) and Scientific Research Publishing Inc. This work is licensed under the Creative Commons Attribution International License (CC BY 4.0).

http://creativecommons.org/licenses/by/4.0/

\section{(c) (i) Open Access}

\begin{abstract}
This investigation was undertaken to enhance the solubility and consequent antibacterial activity of cefuroxime axetil (CA), a $\beta$-lactamase-stable broad spectrum second generation cephalosporin through solid dispersion (SD) technique. For this purpose, CA loaded SDs (CSDs) were prepared by solvent evaporation method using different concentrations of microcrystalline cellulose (MCC) as carrier. The CSDs were characterized by in-vitro dissolution study, thermal analysis (DSC), crystallinity (PXRD), interactions (FTIR) and morphology (SEM). Among the formulations, CSD-2 showed the highest dissolution rate which was 2.59-fold higher than pure CA with a drug-carrier (CA: MCC) ratio of 1:3. Enhanced dissolution rate was attributed to conversion of drug from crystalline to amorphous state during preparation of SDs, which was validated by DSC, PXRD, FTIR and SEM analyses. Antibacterial activity of CSD-2 against Staphylococcus aureus (ATCC 25923) and Escherichia coli (ATCC 25922) showed 1.94- and 6.75-fold higher relative zone of inhibition (RZOI), respectively than pure CA. CSD-2 has been found to be the most effective optimized formulation in terms of both enhanced dissolution rate and antibacterial activity. Thus, it can be an effective alternative to conventional dosage forms of CA. However, further investigations are needed to validate its pharmacokinetic properties, in-vivo antibacterial efficacy and safety before recommending as a novel formulation.
\end{abstract}

\section{Keywords}

Cefuroxime Axetil, Dispersion, Solubility, Antibacterial Activity 


\section{Introduction}

Aqueous solubility of drugs plays a crucial role in determining the rate and extent of its absorption, thus finally affecting its bioavailability. Drugs with poor aqueous solubility are still a challenge in the successful formulation of therapeutic agents. It was reported that $40 \%$ of drugs from newly developed chemical entities are poorly soluble in water, of which $10 \%$ failed to reach the market for their poor aqueous solubility [1]. Oral administration of drug is the most common and preferred route of delivery due to much convenience and patient's compliance. However, inadequate bioavailability is one of the major limiting factors due to poor rate of drug release from solid oral dosage forms, which helps to ascertain the therapeutic efficacy in view of the quantity of drug available for absorption and reaching into blood circulation [2]. So, the venture to improve the solubility and dissolution of poorly water soluble drugs remains one of the delicate tasks in formulation development [3]. A variety of formulation strategies including particle size reduction by micronization, modification of the crystal habit e.g. polymorphs, nanocrystals, drug dispersion in carriers, solid dispersions, change of $\mathrm{pH}$, use of buffer, salt formation, supercritical fluid process, use of adjuvant like surfactants, co-solvents, hydrotropes and novel excipients have been explored to overcome the poor aqueous solubility of drugs [4] [5] [6]. Among these, solid dispersions (SDs) are now firmly recognized as platform technology to improve the bioavailability of poor aqueous soluble drugs by changing particle size distribution, enhancing the wettability \& porosity and polymorphic change apart from its high loading capacity [7] [8]. Of various methods for SDs, the solvent evaporation method that comprises dissolving the drug and carrier in an appropriate solvent, followed by evaporation of the solvent to obtain the expected SD is much convenient for its simplicity [9] [10] [11] [12].

Cefuroxime axetil (CA) is the $\beta$-lactamase-stable acetoxyethyl ester of cefuroxime with a broad spectrum antibacterial activity against both Gram-positive and Gram-negative infections [13]. But being a member of BCS class II drugs, the major drawback is its poor solubility in biological fluids resulting in poor bioavailability after oral administration. It is practically insoluble in water (107 $\mathrm{mg} / \mathrm{L}$ at $25^{\circ} \mathrm{C}$ ) and exhibits low and/or variable bioavailability and hence requires higher oral dose [14]. To enhance the solubility and bioavailability, we have formulated CA loaded solid dispersions (CSDs) using MCC (Avicel PH-102) as carrier in the present investigation. In addition, to validate the clinical implication of optimized CSD formulation, in-vitro antibacterial efficacy testing against both Gram-positive and Gram-negative bacteria was conducted and performance was compared with that of pure drug. Findings of the present investigation are speculated to have pharmacological implications in choosing the appropriate formulation with better clinical efficacy. To the best of our knowledge, it is a unique report on CSD formulation technique and antibacterial efficacy assessment. 


\section{Materials and Methods}

\subsection{Materials}

CA (micronized) and MCC (Avicel PH-102) were generous gift from the Square Pharmaceuticals Ltd., Bangladesh. All other chemicals and solvents used in this work were of analytical grade.

\subsection{Preparation of Solid Dispersions of Cefuroxime Axetil}

Solid dispersion formulations loaded with CA (CSDs) were prepared by solvent evaporation method using MCC as carrier. Briefly, accurately weighed $100 \mathrm{mg}$ of CA was dissolved in sufficient quantity of acetone, which was added into $250 \mathrm{mg}$ of MCC mixed with acetone under continuous stirring by hotplate magnetic stirrer (Wisd, Korea) at $320 \mathrm{rpm}$ to allow sufficient loading of the drug into carrier. This preparation (CSD-1) gave a solid dispersion ratio of 1:2.5 for CA and carrier. The same procedure was followed for making CSD-2 \& CSD-3 with ratios of 1:3 and 1:3.5, respectively by adjusting the volume of carrier (Table 1 ). The temperature was maintained at $56^{\circ} \mathrm{C}$ to evaporate the solvent completely from the dispersion system. When the evaporation of the solvent was completed, the hotplate stirrer was stopped and the CSDs were obtained as dried powder at room temperature. Finally, these were passed through 120 mesh screen to get dried granules of uniform size and stored for further experiments.

\subsection{Dissolution Study}

In-vitro dissolution study was performed for both pure CA and CSDs to demonstrate the release pattern of drug from CSDs in comparison to pure CA according to paddle method (USP Apparatus II) using a dissolution tester (Tianjin Guoming Medicinal Equipment Co. Ltd.) [16] [17]. Demineralised (DM) water was used as dissolution medium. Briefly, sample of CSDs equivalent to $13.5 \mathrm{mg}$ of CA was taken in Fisherbrand dialysis tubing (MW: 12,000 - 14,000; width: 45 mm; wall thickness: $20 \mu \mathrm{m}$; dry cylinder diameter: $28.6 \mathrm{~mm}$; volume/cm: 6.42 ) and immersed in $900 \mathrm{ml}$ of dissolution medium contained in a dissolution vessel. The paddle speed and temperature were maintained at $50 \pm 2 \mathrm{rpm}$ and $37.0^{\circ} \mathrm{C} \pm 0.5^{\circ} \mathrm{C}$, respectively. The analyte samples $(10 \mathrm{ml}$ each $)$ were withdrawn at $1,5,15,30,60,120,150,180,210,240,270,300,360,420,480,540,600,660$ and $720 \mathrm{~min}$ followed by the replacement of an equal volume of fresh dissolution medium, respectively. Concentration of CA at each point was assayed by measuring absorbance at $281 \mathrm{~nm}$ with a UV-spectrophotometer (UV mini-1240, Shimadzu,

Table 1. Drug-carrier ratio of solid dispersion formulations loaded with CA (CSDs) [15].

\begin{tabular}{ccc}
\hline Formulation & Drug \& Carrier & Ratio \\
\hline CSD-1 & CA \& MCC & $1: 2.5$ \\
CSD-2 & CA \& MCC & $1: 3.0$ \\
CSD-3 & CA \& MCC & $1: 3.5$ \\
\hline
\end{tabular}


Japan) using a calibration curve. Three replicates of each sample were carried out and mean values were calculated. The mean concentration of CA was plotted against time to derive the dissolution profile.

\subsection{Solid State Characterization of CSDs}

\subsubsection{Differential Scanning Calorimetry (DSC)}

Thermograms of pure CA, MCC and CSDs were obtained from DSC (Exstar SII DSC7020, Hitachi High-Tech Science Corporation, Tokyo, Japan). Each sample (3 - $5 \mathrm{mg}$ ) was placed in sealed standard aluminium pans and heated from $0^{\circ} \mathrm{C}$ $300^{\circ} \mathrm{C}$, at a scanning rate of $10^{\circ} \mathrm{C} / \mathrm{min}$ under nitrogen purge with an empty aluminium pans as reference [18].

\subsubsection{Powder X-Ray Diffraction (PXRD)}

CA, MCC and CSDs were analysed by PXRD. An X-ray diffractometer (RAD-C, Rigaka Denki Co. Ltd. Tokyo, Japan) was used for the diffraction studies. The samples were exposed to $\mathrm{Cu}-\mathrm{K} \alpha$ radiation $(30 \mathrm{kV}$ and $50 \mathrm{~mA})$ and scanned from $2^{\circ}-40^{\circ}, 2 \theta$ at a scanning rate of $5^{\circ} / \mathrm{min}[18]$.

\subsubsection{Fourier Transform Infrared Spectroscopy (FTIR)}

The nature of drug-carrier interactions in the CSDs were investigated using IR spectra measured by the diffuse reflection method using an FTIR spectrometer (IR-Prestige 21, Shimadzu Co. Japan). Disk of samples were prepared by grounding and thorough mixing with potassium bromide. The scanning range was 400 to $4000 \mathrm{~cm}^{-1}$ and the resolution was $1 \mathrm{~cm}^{-1}$ [18].

\subsubsection{Scanning Electron Microscopy (SEM)}

The shape, surface and cross-sectional morphology of pure CA, MCC and CSDs were observed using a scanning electron microscope (SSX-500, Shimadzu, Japan) after platinum metallization. An accelerating voltage of $15 \mathrm{kV}$ was used [18].

\subsection{Antibacterial Activity of Pure CA and CSD-2 Formulation}

\subsubsection{Source of Bacteria}

Staphylococcus aureus (ATCC 25923) and Escherichia coli (ATCC 25922) were used for antibacterial activity testing of pure CA and CSD-2 formulation. ATCC bacterial strains were collected from the Microbiology Department of Rajshahi Medical College, Bangladesh.

\subsubsection{Preparation of Antimicrobial Disks of CSD-2}

From dissolution vessel, $10 \mathrm{ml}$ aliquot of CSD-2 formulation were withdrawn at $60,120,180,240,300,360,420,480,540,600,660$ and $720 \mathrm{~min}$, respectively and separately extracted with $5 \mathrm{ml}$ of chloroform. The extraction process was repeated for 3 times and extracted samples were kept in vials for drying. After being completely dried, $300 \mu \mathrm{l}$ of acetone were added into each vial and the vials were shaken vigorously to ensure complete solution. The solution was applied to a sterilized blank disk (Bio Maxima SA Poland) kept into a sterilized Petri dish 
so that the theoretical concentration of CA reaches to $30 \mu \mathrm{g} / \mathrm{disk}$. The disks were made readily available for antibacterial testing when they were dried completely after evaporation of acetone. Three replicates of each sample were carried out.

\subsubsection{Antibacterial Activity Testing}

Disk diffusion method as described by Kirby-Bauer was applied to determine the antibacterial efficacy of CSD-2 formulation that showed the highest dissolution profile among CSD formulations. Colonies from fresh bacterial culture of both $S$. aureus and $E$. coli were inoculated into $5 \mathrm{ml}$ of $0.9 \%$ saline solution and turbidity was matched with $0.5 \mathrm{McF}$ arland standards to optimize inoculation at a concentration of $10^{7}$ colony-forming units (CFU)/ml. $100 \mu \mathrm{l}$ of inoculum were spread gently onto the freshly prepared Mueller-Hinton agar medium with sterilized cotton buds. Before using the plates for inoculation, care was taken to ensure that the plate was free from accumulated moisture on the lid or surface of the agar. For the test, $5 \mu \mathrm{l}$ aliquots of each culture were placed on the agar plates. The controls on each plate were the bacterial inoculum without CA added for determining the bacterial growth and medium for sterility control. Then antimicrobial disks prepared at different time period were placed onto the inoculated medium following standard technique and was kept into refrigerator for 3 hours for ensuring adequate diffusion. To compare the zone of inhibition (ZOI), a reference disk prepared from pure drug (CA) was placed at the centre of the medium and kept into incubator at $37^{\circ} \mathrm{C}$ overnight. The ZOI around each disk was measured in $\mathrm{mm}$ [19]. The mean value of ZOI was calculated from three replicates.

\subsubsection{Determination of Relative Zone of Inhibition (RZOI)}

The RZOI was determined by calculating the diameters of each ZOI of samples divided by diameter of control disk on the same Petri dish using the following equation:

$$
\mathrm{RZOI}=\frac{\text { ZOIs }}{\text { ZOIc }}
$$

where, RZOI = Relative zone of inhibition; ZOIs = Diameter of ZOI of sample; ZOIc $=$ Diameter of ZOI of control.

\subsection{Statistical Analysis}

Results were expressed as mean \pm standard deviation (S.D.). Differences among groups were assessed by unpaired $t$-test using GraphPad Prism 8.0.1. Values of $\mathrm{p}$ $<0.05$ were considered statistically significant.

\section{Results}

\subsection{Dissolution Study}

Dissolution profiles of various CSDs and pure CA are depicted in Table 2 and Figure 1. All three formulations showed improvement in drug dissolution with CSD-1, CSD-2 and CSD-3 had 1.66, 2.60 and 2.48-fold higher dissolution rate, 
Table 2. Dissolution profiles of solid dispersions loaded with CA.

\begin{tabular}{|c|c|c|c|c|}
\hline \multirow{2}{*}{$\begin{array}{l}\text { Time } \\
(\min )\end{array}$} & \multicolumn{4}{|c|}{ Concentration $(\mu \mathrm{g} / \mathrm{ml})$} \\
\hline & CA & CSD-1 & $\mathrm{CSD}-2$ & $\mathrm{CSD}-3$ \\
\hline 1 & $0.30 \pm 0.050$ & $0.32 \pm 0.025^{c}$ & $0.50 \pm 0.017^{\star *}$ & $0.38 \pm 0.014^{c}$ \\
\hline 5 & $0.51 \pm 0.058$ & $0.54 \pm 0.014^{\mathrm{b}}$ & $0.68 \pm 0.029^{*}$ & $0.57 \pm 0.028^{\mathrm{b}}$ \\
\hline 15 & $1.03 \pm 0.076$ & $0.80 \pm 0.049^{\star \mathrm{b}}$ & $1.04 \pm 0.034$ & $0.98 \pm 0.025$ \\
\hline 30 & $1.43 \pm 0.029$ & $1.02 \pm 0.028^{\star * * c}$ & $2.01 \pm 0.045^{\star \star \star}$ & $1.76 \pm 0.038^{\star * \star b}$ \\
\hline 60 & $2.04 \pm 0.086$ & $1.82 \pm 0.043^{* c}$ & $3.91 \pm 0.051^{\star * *}$ & $3.20 \pm 0.049^{* * * c}$ \\
\hline 90 & $2.74 \pm 0.050$ & $2.62 \pm 0.038^{\star c}$ & $5.25 \pm 0.061^{\star \star *}$ & $4.38 \pm 0.043^{\star * \star c}$ \\
\hline 120 & $3.12 \pm 0.160$ & $3.24 \pm 0.057^{\mathrm{c}}$ & $6.52 \pm 0.068^{\star * *}$ & $5.52 \pm 0.057^{* * * c}$ \\
\hline 150 & $3.75 \pm 0.125$ & $3.78 \pm 0.062^{c}$ & $7.79 \pm 0.078^{\star * *}$ & $6.54 \pm 0.065^{\star * * c}$ \\
\hline 180 & $4.24 \pm 0.132$ & $4.54 \pm 0.051^{\star c}$ & $8.95 \pm 0.085^{\star * *}$ & $7.39 \pm 0.051^{* * * c}$ \\
\hline 210 & $4.63 \pm 0.086$ & $5.04 \pm 0.074^{* * * c}$ & $9.79 \pm 0.059^{\star * *}$ & $8.38 \pm 0.062^{\star * * c}$ \\
\hline 240 & $5.13 \pm 0.100$ & $5.52 \pm 0.075^{* * c}$ & $10.61 \pm 0.09^{* * *}$ & $9.23 \pm 0.086^{* * * c}$ \\
\hline 270 & $5.55 \pm 0.104$ & $6.07 \pm 0.086^{* * c}$ & $11.35 \pm 0.102^{* * *}$ & $9.84 \pm 0.014^{* * * c}$ \\
\hline 300 & $5.86 \pm 0.152$ & $6.61 \pm 0.089^{* * c}$ & $12.17 \pm 0.017^{\star * *}$ & $10.53 \pm 0.038^{* * * c}$ \\
\hline 360 & $5.96 \pm 0.125$ & $7.60 \pm 0.025^{\star * * c}$ & $13.40 \pm 0.119^{\star * *}$ & $11.94 \pm 0.079^{* * * c}$ \\
\hline 420 & $6.03 \pm 0.050$ & $8.19 \pm 0.049^{* * * c}$ & $14.26 \pm 0.106^{* * *}$ & $12.79 \pm 0.025^{* * * c}$ \\
\hline 480 & $6.05 \pm 0.076$ & $8.70 \pm 0.038^{* * * c}$ & $15.10 \pm 0.061^{\star * *}$ & $13.70 \pm 0.051^{* * * c}$ \\
\hline 540 & $6.03 \pm 0.100$ & $9.21 \pm 0.014^{* * * c}$ & $15.75 \pm 0.136^{\star * *}$ & $14.43 \pm 0.062^{* * * c}$ \\
\hline 600 & $6.03 \pm 0.115$ & $9.68 \pm 0.079^{* * * c}$ & $15.79 \pm 0.051^{\star * *}$ & $14.84 \pm 0.086^{* * * c}$ \\
\hline 660 & $6.05 \pm 0.144$ & $10.00 \pm 0.057^{* * * c}$ & $15.73 \pm 0.090^{\star * *}$ & $14.94 \pm 0.057^{* * * c}$ \\
\hline 720 & $6.05 \pm 0.058$ & $10.02 \pm 0.028^{* * * c}$ & $15.77 \pm 0.061^{\star * *}$ & $14.97 \pm 0.079^{* * * c}$ \\
\hline
\end{tabular}

Results are presented as mean \pm S.D. $(\mathrm{n}=3){ }^{*} \mathrm{p}<0.05,{ }^{* *} \mathrm{p}<0.01,{ }^{* *} \mathrm{p}<0.001$ vs. CA and ${ }^{\mathrm{a}} \mathrm{p}<0.05,{ }^{\mathrm{b}} \mathrm{p}<$ $0.01,{ }^{c} \mathrm{p}<0.001$ vs CSD -2 .

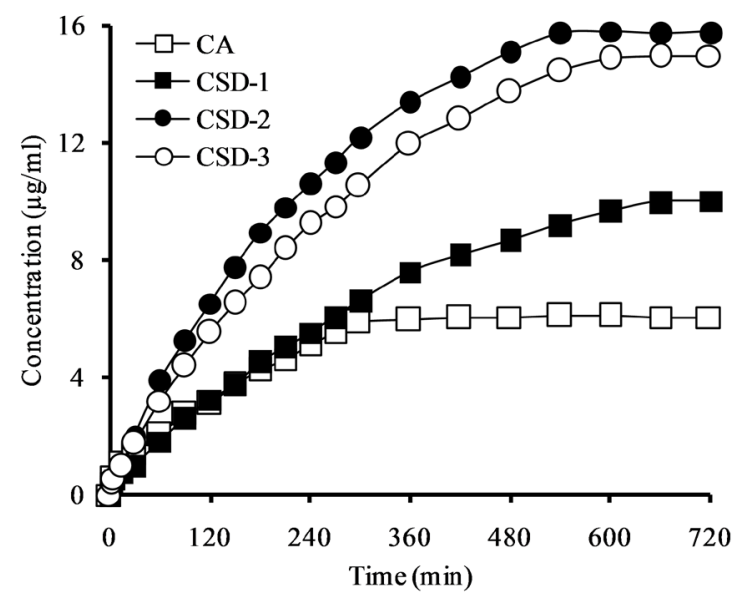

Figure 1. Dissolution profile of CA, CSD-1, CSD-2 and CSD-3. Data of Table 2 are shown in this figure. Each value represents mean \pm S.D. $(n=3)$. Statistical analyses are shown in Table 2. 
respectively than that of pure CA. The peak drug concentration produced by CA was $6.05 \mu \mathrm{g} / \mathrm{ml}$ obtained at $480 \mathrm{~min}$. In comparison, the same concentration was noted in case of CSD- 1 at $270 \mathrm{~min}, \mathrm{CSD}-2$ at $120 \mathrm{~min}$ and CSD-3 at $150 \mathrm{~min}$, demonstrating that all formulations were capable of faster drug release in water than CA. Further, the peak concentrations produced by CSD-1, CSD-2 and CSD-3 were $10.02,15.79$ and $14.97 \mu \mathrm{g} / \mathrm{ml}$ at 720,600 and $720 \mathrm{~min}$, respectively. Among the formulations, CSD-2 was found to produce significantly higher ( $\mathrm{p}<$ 0.001) drug concentration in comparison to CSD-1 and CSD-3 (Table 2). Therefore, CSD-2 was considered as the optimum formulation for its enhanced drug release profile with a drug carrier ratio of 1:3 (CA: MCC) and was selected for further antibacterial activity study.

\subsection{DSC Study}

The thermal stability of CSD formulations was evaluated using DSC. It gives an insight into the melting and re-crystalline behaviour of crystalline materials. Therefore, DSC experiments are useful to understand the crystallinity properties of CSDs. Thermal characteristics of CA and different CSDs are presented in Figure 2. Being micronized CA generated an sharp endothermic peak with low intensity, corresponding to its melting point $(\mathrm{mp})$ of $82^{\circ} \mathrm{C}$ indicating partial crystallinity of the drug. The carrier MCC showed a wide mp peak at $85^{\circ} \mathrm{C}$. But CSD-1, CSD-2 and CSD-3 showed no mp peak for CA except broad endothermic peaks for MCC. These results demonstrate the possibility of conversion of CA from crystalline to amorphous state during preparation of the solid dispersions.

\subsection{PXRD Study}

Figure 3 shows the X-ray diffraction patterns of CA (micronized), MCC and CSDs. The PXRD pattern of CA showed absence of significant sharp peaks due to micronization of the drug that was also concordant with its DSC thermogram.

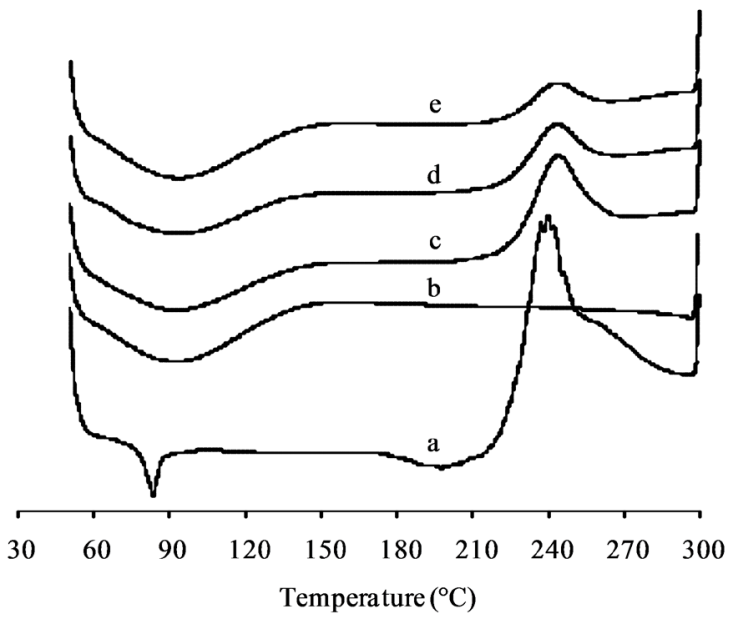

Figure 2. DSC thermograms of (a) CA; (b) MCC; (c) CSD-1; (d) CSD-2; (e) CSD-3. 


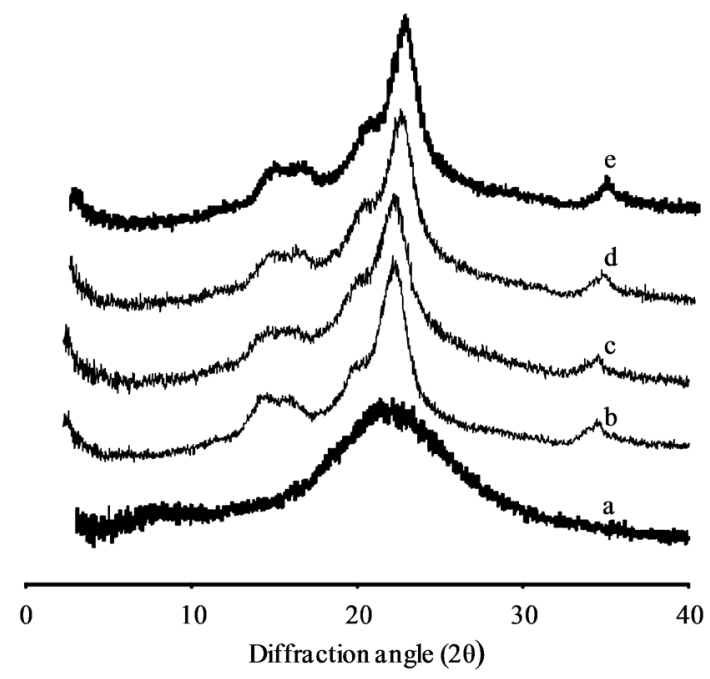

Figure 3. PXRD patterns of (a) CA; (b) MCC; (c) CSD-1; (d) CSD-2; (e) CSD-3.

In X-ray powder diffractograms, CA showed only a broad peak with intensity at $2 \theta$ scattered angle $21.48^{\circ}$. Whereas the carrier, MCC exhibited a broaden peak at $22.50^{\circ}$ and a small peak at $34.40^{\circ}$ which persisted in CSD- $1\left(22.42^{\circ}, 34.28^{\circ}\right)$; CSD-2 $\left(22.56^{\circ}, 34.46^{\circ}\right)$ and CSD-3 $\left(22.6^{\circ}, 34.38^{\circ}\right)$ formulations too. However, there was no visible peak for CA in the CSDs except the peaks for MCC. Similar to DSC analysis, PXRD also demonstrated about the conversion of CA to amorphous state.

\subsection{FTIR Study}

FTIR spectra of the optimized CSDs were studied to describe the possible interactions between CA and carrier (MCC) during preparation of solid dispersion. The results of FTIR spectroscopy of CA, MCC and CSDs are shown in Figure 4.

The spectrum of CA was characterized by peaks at $3480-3210 \mathrm{~cm}^{-1}$ (for $\mathrm{NH}$, $\mathrm{NH}_{2}$ complex), $1782 \mathrm{~cm}^{-1}$ (for $\beta$-lactam), $1760 \mathrm{~cm}^{-1}$ (for acetate), $1720 \mathrm{~cm}^{-1}$ (for 4-ester group), 1676 and $1534 \mathrm{~cm}^{-1}$ (for 7-amido) [15]. The FTIR spectrum of MCC exhibited O-H stretching absorption around $3449 \mathrm{~cm}^{-1}$, C-H stretching absorption around $2900-3000 \mathrm{~cm}^{-1}$ and C-O-C stretching absorptions around 1061 and $1104 \mathrm{~cm}^{-1}$, respectively [20].

However, the stretching band intensity by $\mathrm{C}-\mathrm{O}-\mathrm{C}, \mathrm{O}-\mathrm{H}$ and $\mathrm{C}-\mathrm{H}$ of $\mathrm{MCC}$ was reduced in CSD-1, CSD-2 and CSD-3 in FTIR spectra. The bands due to $\beta$-lactam, esters, and amides were totally disappeared in all the formulations. Due to presence of O-H groups in the structure of MCC a lot, there was much possibility to form weak hydrogen bonds with polar part of $\beta$-lactam, ester and amide groups of CA resulting in decreased bond energy to break down and thus responsible for increasing wettability of the conjugates. This evidence also suggested a possibility of chemisorption of CA on MCC structure forming weak hydrogen bond that might have facilitated for enhanced dissolution rate of the drug. 


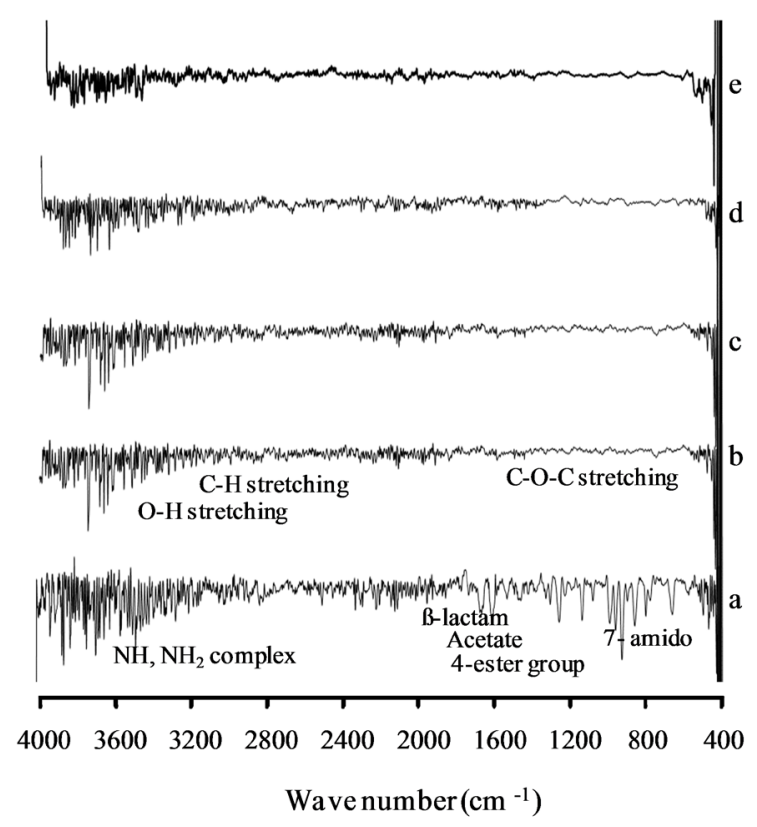

Figure 4. FTIR spectra of (a) CA; (b) MCC; (c) CSD-1; (d) CSD-2; (e) CSD-3.

\subsection{SEM Study}

The SEM images of CA, MCC and CSDs are shown in Figure 5. In the micrograph, CA (Figure 5(a)) appeared as regular spherical particles with uniform and smooth surfaces. MCC (Figure 5(b)) exhibited an irregular shaped crystal. But in the micrographs of CSD-1, CSD-2 and CSD-3 (Figures 5(c)-(e)) no particle of CA was observed indicating adsorption of CA molecules on the surfaces of MCC might have taken place. According to FTIR results these adsorptions occurred due to possibility of H-bonding among the O-H/C-H groups of CA and O-H groups of MCC. Further, among these formulations, CSD-2 (Figure 5(d)) exhibited some network like structure ("X" marked) that facilitated prompt water absorption resulting in quick and enhanced dissolution of CA.

Taken together, DSC, PXRD, and SEM images indicated that the crystalline behaviour of CA was eliminated at various degrees in the CSDs by using MCC. The possible reason for this conversion might be chemisorption of CA on the surface of MCC molecules through weak H-bonding between -O-H groups of MCC and the exhibited functional groups of the drug. The enhanced drug release profile from CSDs might be the result of such interaction mediated chemisorption at various extents.

Among the CSDs, CSD-2 can be designated as optimum solid dispersion formulation considering its dissolution behaviour, DSC, PXRD, FTIR and SEM data.

\subsection{In-Vitro Antibacterial Activity of Pure CA and CSD-2}

The optimized CSD (CSD-2) and pure CA were selected for in-vitro antibacterial activity testing against both Gram positive (S. aureus, ATCC 25923) and Gram negative (E. coli, ATCC 25922) bacteria. 


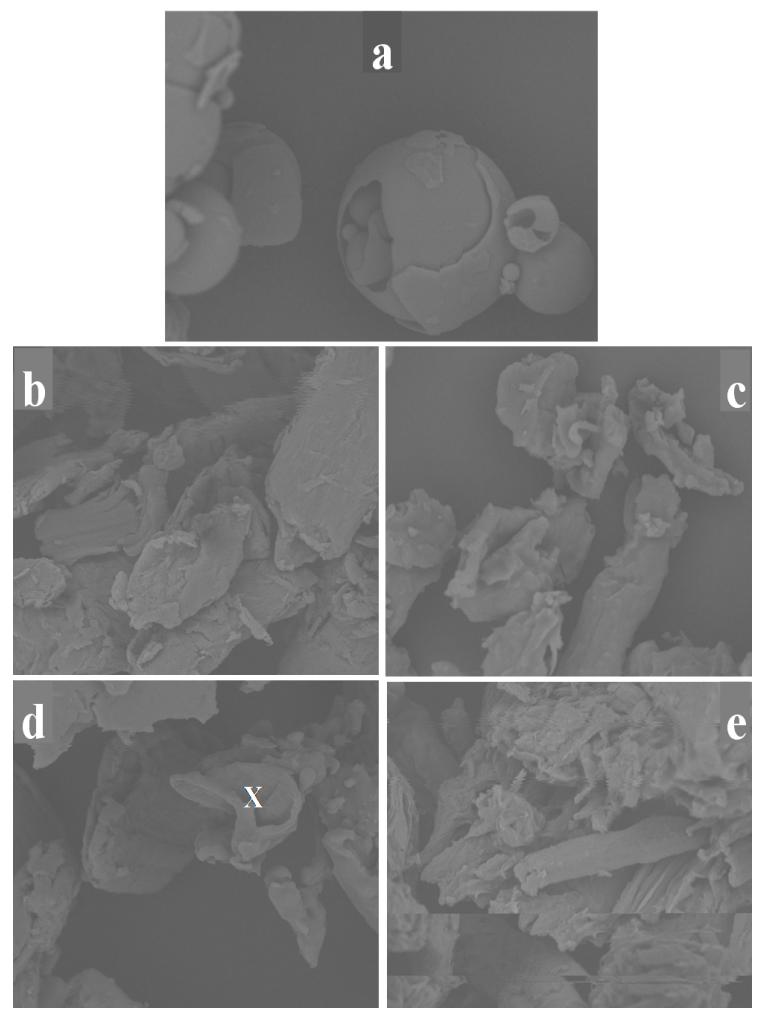

Figure 5. SEM images ( $\times 1.0 \mathrm{k})$; (a) CA; (b) MCC; (c) CSD-1; (d) CSD-2; (e) CSD-3.

\subsubsection{Relative Zone of Inhibition of CSD-2 against $S$. aureus}

In antibacterial activity testing, there was gradual increase in relative zone of inhibition (RZOI) of CSD-2 than pure CA against $S$. aureus (Figure 6).

The increased RZOI produced by CSD-2 were statistically highly significant ( $\mathrm{p}$ $<0.001$ ) up to 300 min but significance levels varied between $\mathrm{p}<0.001$ to $\mathrm{p}<$ 0.05 in comparison to pure CA.

\subsubsection{Relative Zone of Inhibition of CSD-2 against $E$. coli}

Parallel to $S$. aureus, RZOI produced by CSD-2 against $E$. coli also showed gradual increase than pure CA with time in antibacterial activity testing (Figure 7).

The increased RZOI produced by CSD-2 were statistically highly significant ( $\mathrm{p}$ $<0.001$ ) up to $540 \mathrm{~min}$ but significance levels varied between $\mathrm{p}<0.001$ to $\mathrm{p}<$ 0.05 in comparison to pure CA.

\section{Discussion}

Cefuroxime axetil is a BCS class II drug having very poor aqueous solubility that plays a limiting role on its bioavailability [21]. To overcome this limitation solid dispersion formulations were developed to enhance the aqueous solubility and hence bioavailability of this hydrophobic drug. For this, CSDs were prepared using MCC (Avicel PH-102) as carrier. The resultant CSD formulations were characterized and evaluated through in-vitro dissolution, solid state characterization by DSC, PXRD, FTIR and SEM and in-vitro antibacterial efficacy study. 


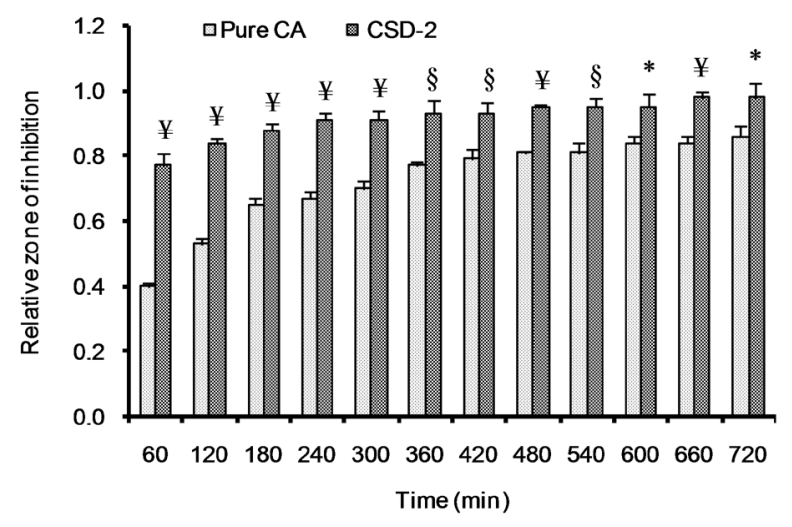

Figure 6. Relative zone of inhibition of pure CA and CSD-2 against $S$. aureus. Results are presented as the mean \pm S.D. $(\mathrm{n}=3)$ with ${ }^{*} \mathrm{p}<0.05,{ }^{\mathrm{s}} \mathrm{p}<0.01,{ }^{\mathrm{x}} \mathrm{p}<0.001$ vs CA.

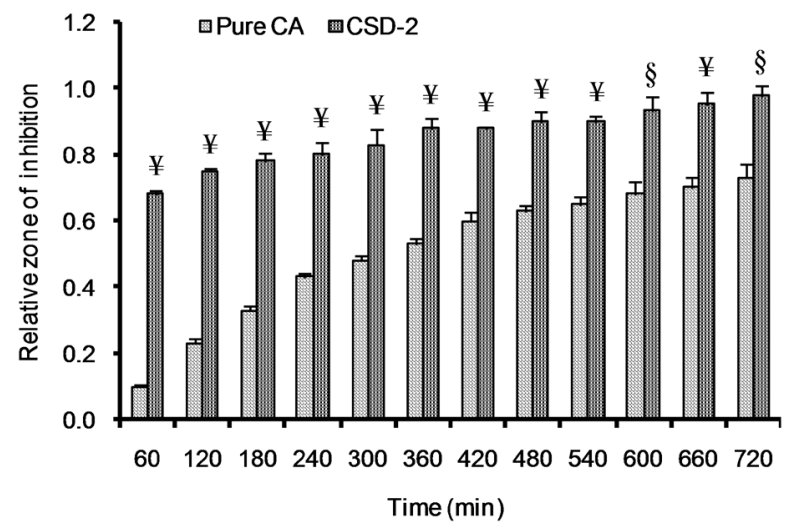

Figure 7. Relative zone of inhibition of pure CA and CSD-2 against $E$. coli. Results are presented as the mean \pm S.D. (n $=3$ ) with ${ }^{\star} \mathrm{p}<0.05,{ }^{\S} \mathrm{p}<0.01,{ }^{\sharp} \mathrm{p}<0.001$ vs CA.

In dissolution study, maximum release of the drug obtained from CSD-2 $(15.79 \mu \mathrm{g} / \mathrm{ml})$ was 2.59 -fold higher than that of pure CA at $600 \mathrm{~min}$ (Table 2 and Figure 1). This improved dissolution of CA was due to increased hydrophilicity achieved through preparation of CSDs. This was mainly attributed to chemisorption of drug on MCC and increased wettability of MCC that was authenticated by physico-chemical characterization.

DSC thermograms (Figure 2) of the CSDs proved the conversion of the drug from crystalline to amorphous form which was also demonstrated by PXRD data (Figure 3). The other mechanism, such as physical bonding between the carriers and drugs were demonstrated by FTIR spectrum (Figure 4), suggesting that this interaction might have improved the wettability properties of the drug and further increased drug dissolution rates [22] [23] [24].

Further, SEM photographs (Figure 5) showed the particle size and surface morphology of the CSDs. The surface morphology studies revealed adsorption of CA molecules on the surfaces of MCC that also might have contributed to the enhancement of the dissolution rate of CA in CSD-1, CSD-2 and CSD-3. 
In literature review we didn't find any work reporting CA loaded solid dispersions using MCC as carrier for enhancing solubility and bioavailability of CA. So, it is not possible to compare and contrast our findings exactly with that of others. But in similar works Gorajana et al. (2015) and Arora et al. (2010) reported that dissolution rate of CA was increased by preparing solid dispersion using hydrophilic carrier (PVP K30/PEG 4000) and urea, respectively through the solvent evaporation method [15] [25]. In another report, Sankari and Al-Hariri (2018) also described that solubility and dissolution rate of CA can be improved significantly by preparing solid dispersions through different methods including solvent evaporation technique using water soluble carrier poloxamer 188 [26]. Although these researches used different carrier than ours but as far as the dispersion technique and its outcomes are concerned, these reports are found to be consistent with the findings of the present study that solid dispersion formulations using carrier showed significant increase in dissolution rate and aqueous solubility of CA with resultant enhanced bioavailability.

In-vitro antibacterial activity testing showed CSD-2 had significantly higher ZOI than pure CA. The RZOI of CSD-2 formulation was 1.94 and 6.75-fold higher than pure CA at $60 \mathrm{~min}$ against $S$. aureus and $E$. coli, respectively (Figure 6 \& Figure 7). The statistical analysis revealed that there was significant difference $(\mathrm{p}<0.001)$ of RZOI between pure CA and CSD-2. It can be correlated with the fact that the enhanced solubility of CA with resultant better diffusion achieved through CSD formulation attributed for significant difference in RZOI.

Khan et al. (2016) reported that, cefixime nanoparticles exhibited better antibacterial activity than pure cefixime against $S$. aureus and $E$. coli by agar well diffusion method [27]. Omolo et al. (2018) demonstrated that antibacterial activities of different nanosuspension formulations of fusidic acid delivered through poloxamer 188 were found better as compared to pure fucidic acid [28]. Further, it was revealed that both in-vitro and in-vivo test of CA amorphous ultrafine particles by high gravity anti-solvent precipitation (HGAP) technique possessed better dissolution rate and stronger antibacterial activity by agar dilution method against $S$. aureus and E. coli [29].

Although dispersion technique and drug are different in the present investigation but reports pertaining to antibacterial efficacy of formulations prepared through various methods mentioned above suggest that formulations had reportedly significantly better antibacterial efficacy over pure drug which corroborates with our results.

\section{Conclusion}

Cefuroxime axetil loaded solid dispersion using MCC as a carrier was found to be an improved formulation technique in terms of both dissolution rate and antibacterial activity of CA. Among the formulations, CSD-2 was found to be the best and this newer formulation of CA can be an effective alternative to its conventional oral dosage forms. However, further investigations are needed to vali- 
date its in-vivo pharmacokinetics, antibacterial efficacy and safety before being its acceptance as a novel formulation.

\section{Acknowledgements}

The authors are thankful to Square Pharmaceuticals Ltd., Bangladesh for providing Cefuroxime Axetil and excipients used in this research work.

\section{Funding}

This research work was funded by Ministry of Science and Technology, Government of the People's Republic of Bangladesh.

\section{Conflicts of Interest}

The authors declare that there is no conflict of interests.

\section{References}

[1] Kalepu, S. and Nekkanti, V. (2015) Insoluble Drug Delivery Strategies: Review of Recent Advances and Business Prospects. Acta Pharmaceutica Sinica B, 5, 442-453. https://doi.org/10.1016/j.apsb.2015.07.003

[2] Homayun, B., Lin, X.T. and Choi, H.-J. (2019) Challenges and Recent Progress in Oral Drug Delivery Systems for Biopharmaceuticals. Pharmaceutics, 11, 129. https://doi.org/10.3390/pharmaceutics11030129

[3] Tran, P., Pyo, Y.-C., Kim, D.-H., Lee, S.-E., Kim, J.-K. and Park, J.-S. (2019) Overview of the Manufacturing Methods of Solid Dispersion Technology for Improving the Solubility of Poorly Water-Soluble Drugs and Application to Anticancer Drugs. Pharmaceutics, 11, 132. https://doi.org/10.3390/pharmaceutics11030132

[4] Savjani, K.T., Gajjar, A.K. and Savjani, J.K. (2012) Drug Solubility: Importance and Enhancement Techniques. ISRN Pharmaceutics, 2012, Article ID: 195727.

https://doi.org/10.5402/2012/195727

[5] Singh, A., Worku, Z.A. and Van Den Mooter, G. (2011) Oral Formulation Strategies to Improve Solubility of Poorly Water-Soluble Drugs. Expert Opinion on Drug Delivery, 8, 1361-1378. https://doi.org/10.1517/17425247.2011.606808

[6] Huang, Y. and Dai, W.G. (2014) Fundamental Aspects of Solid Dispersion Technology for Poorly Soluble Drugs. Acta Pharmaceutica Sinica B, 4, 18-25. https://doi.org/10.1016/j.apsb.2013.11.001

[7] Thenmozhi, K. and Yoo, Y.J. (2017) Enhanced Solubility of Piperine Using Hydrophilic Carrier-Based Potent Solid Dispersion Systems. Drug Development and Industrial Pharmacy, 43, 1501-1509. https://doi.org/10.1080/03639045.2017.1321658

[8] Praveen, K. and Chhater, S. (2013) A Study on Solubility Enhancement Methods for Poorly Water Soluble Drugs. American Journal of Pharmacological Sciences, 1, 67-73. https://doi.org/10.12691/ajps-1-4-5

[9] Choi, J.S., Lee, S.E., Jang, W.S., Byeon, J.C. and Park, J.S. (2018) Solid Dispersion of Dutasteride Using the Solvent Evaporation Method: Approaches to Improve Dissolution Rate and Oral Bioavailability in Rats. Materials Science and Engineering: $C$, 90, 387-396. https://doi.org/10.1016/j.msec.2018.04.074

[10] Leuner, C. and Dressman, J. (2000) Improving Drug Solubility for Oral Delivery Using Solid Dispersions. European Journal of Pharmaceutics and Biopharmaceu- 
tics, 50, 47-60. https://doi.org/10.1016/S0939-6411(00)00076-X

[11] Lavra, Z.M.M., Pereira De Santana, D. and Re, M.I. (2017) Solubility and Dissolution Performances of Spray-Dried Solid Dispersion of Efavirenz in Soluplus. Drug Development and Industrial Pharmacy, 43, 42-54. https://doi.org/10.1080/03639045.2016.1205598

[12] Verma, V., Sharma, P., Sharma, J., Lamba, A.K. and Lamba, H.S. (2017) Development, Characterization and Solubility Study of Solid Dispersion of Quercetin by Solvent Evaporation Method. Materials Today: Proceedings, 4, 10128-10133. https://doi.org/10.1016/j.matpr.2017.06.334

[13] Mizera, M., Szymanowska, D., Stasiłowicz, A., Siakowska, D., Lewandowska, K., Miklaszewski, A., Plech, T., Tykarska, E. and Cielecka-Piontek, J. (2020) Computer-Aided Design of Cefuroxime Axetil/Cyclodextrin System with Enhanced Solubility and Antimicrobial Activity. Biomolecules, 10, 24.

https://doi.org/10.3390/biom10010024

[14] Ali, A.M.A., Khames, A., Alrobaian, M.M., Hamaidi, M. and Abourehab, M.A.S. (2018) Glucosamine-Paracetamol Spray-Dried Solid Dispersions with Maximized Intrinsic Dissolution Rate, Bioavailability and Decreased Levels of in Vivo Toxic Metabolites. Drug Design, Development and Therapy, 2018, 3071-3084. https://doi.org/10.2147/DDDT.S176099

[15] Gorajana, A., Rajendran, A., Yew, L.M. and Dua, K. (2015) Preparation and Characterization of Cefuroxime Axetil Solid Dispersions Using Hydrophilic Carriers. International Journal of Pharmaceutical Investigation, 5, 171-178. https://doi.org/10.4103/2230-973X.160857

[16] Dhumal, R.S., Biradar, S.V., Aher, S. and Paradkar, A.R. (2009) Cefuroxime Axetil Solid Dispersion with Polyglycolized Glycerides for Improved Stability and Bioavailability. Journal of Pharmacy and Pharmacology, 61, 743-751. https://doi.org/10.1211/jpp.61.06.0006

[17] Rao, K.V. and Venkatchalam, V.V. (2016) Mucoadhesive Biphasic Mini Tablets of Cefuroxime Axetil: Formulation Development, Characterization and in Vivo Bioavailability Study. Journal of Drug Delivery Science and Technology, 35, 260-271. https://doi.org/10.1016/j.jddst.2016.07.003

[18] Barman, R.K., Iwao, Y., Funakoshi, Y., Ranneh, A., Noguchi, S., Wahed, M.I.I. and Itai, S. (2014) Development of Highly Stable Nifedipine Solid-Lipid Nanoparticles. Chemical and Pharmaceutical Bulletin, 62, 399-406. https://doi.org/10.1248/cpb.c13-00684

[19] Bauer, A.W., Kirby, W.M.M., Sherris, J.C. and Turck, M. (1966) Antibiotic Susceptibility Testing by a Standardized Single Disk Method. American Journal of Clinical Pathology, 45, 493-496. https://doi.org/10.1093/ajcp/45.4_ts.493

[20] Setu, M.N., Mia, M.Y., Lubna, N.J. and Chowdhury, A.A. (2014) Preparation of Microcrystalline Cellulose from Cotton and Its Evaluation as Direct Compressible Excipient in the Formulation of Naproxen Tablets. Dhaka University Journal of Pharmaceutical Sciences, 13, 187-192. https://doi.org/10.3329/dujps.v13i2.21899

[21] Sruti, J., Patra, C.N., Swain, S.K., Beg, S., Palatasingh, H.R. and Dinda, S.C. (2013) Improvement in Dissolution Rate of Cefuroxime Axetil by Using Poloxamer 188 and Neusilin US2. Indian Journal of Pharmaceutical Sciences, 75, 67-75. https://doi.org/10.4103/0250-474X.113551

[22] Yin, L.F., Huang, S.J., Zhu, C.L., Zhang, S.H., Zhang, Q., Chen, X.J. and Liu, Q.W. (2012) In-Vitro and in Vivo Studies on a Novel Solid Dispersion of Repaglinide Using Polyvinyl Polyvinylpyrrolidone as the Carrier. Drug Development and In- 
dustrial Pharmacy, 38, 1371-1380. https://doi.org/10.3109/03639045.2011.652635

[23] Patel, J.R., Carlton, R.A., Yuniatine, F., Needham, T.E., Wu, L. and Vogt, F.G. (2012) Preparation and Structural Characterization of Amorphous Spray-Dried Dispersions of Tenoxicam with Enhanced Dissolution. Journal of Pharmaceutical Sciences, 101, 641-663. https://doi.org/10.1002/jps.22800

[24] Pahovnik, D., Reven, S., Grdadolnik, J., Mavri, J. and Zagar, E. (2011) Determination of the Interaction between Glimepiride and Hyperbranched Polymers in Solid Dispersions. Journal of Pharmaceutical Sciences, 100, 4700-4709. https://doi.org/10.1002/jps.22662

[25] Arora, S.C., Sharma, P.K., Irchhaiya, R., Khatkar, A., Singh, N. and Gagoria, J. (2010) Development, Characterization and Solubility Study of Solid Dispersions of Cefuroxime Axetil by the Solvent Evaporation Method. Journal of Advanced Pharmaceutical Technology \& Research, 1, 326-329.

https://doi.org/10.4103/0110-5558.72427

[26] Sankari, T. and Al-Hariri, S. (2018) Preparation and Characterization of Cefuroxime Axetil Solid Dispersions Using Poloxamer 188. Brazilian Journal of Pharmaceutical Sciences, 54, e17644. https://doi.org/10.1590/s2175-97902018000417644

[27] Khan, F.A., Zahoor, M., Islam, N.U. and Hameed, R. (2016) Synthesis of Cefixime and Azithromycin Nanoparticles: An Attempt to Enhance Their Antimicrobial Activity and Dissolution Rate. Journal of Nanomaterials, 2016, Article ID: 6909085. https://doi.org/10.1155/2016/6909085

[28] Omolo, C.A., Kalhapure, R.S., Agrawal, N., Rambharose, S., Mocktar, C. and Govender, T. (2018) Formulation and Molecular Dynamics Simulations of a Fusidic Acid Nanosuspension for Simultaneously Enhancing Solubility and Antibacterial Activity. Molecular Pharmacology, 15, 3512-3526. https://doi.org/10.1021/acs.molpharmaceut.8b00505

[29] Zhao, H., Kang, X.L., Chen, X.L., Wang, J.X., Le, Y. and Shen, Z.G. (2009) Antibacterial Activities of Amorphous Cefuroxime Axetil Ultrafine Particles Prepared by High Gravity Anti-Solvent Precipitation (HGAP). Pharmaceutical Development and Technology, 14, 485-491. https://doi.org/10.1080/10837450902762991 